\title{
Boundary Layer Flow of a Nanofluid Through a Permeable Medium Due to Porous Plate
}

\author{
Zahida Khan, Abdul Rehman, Naveed Sheikh, Saleem Iqbal, Ejaz Shah
}

Department of Mathematics, University of Balochistan, Quetta, Pakistan

Email address:

abdul_maths@yahoo.com (A. Rehman)

\section{To cite this article:}

Zahida Khan, Abdul Rehman, Naveed Sheikh, Saleem Iqbal, Ejaz Shah. Boundary Layer Flow of a Nanofluid Through a Permeable Medium Due to Porous Plate. American Journal of Mathematical and Computer Modelling. Vol. 5, No. 4, 2020, pp. 97-101.

doi: 10.11648/j.ajmcm.20200504.11

Received: September 14, 2020; Accepted: October 13, 2020; Published: October 23, 2020

\begin{abstract}
In the present article, an attempted have been made to study the behavior of boundary layer viscous fluid flow and heat transfer containing some nanosized solid particles flowing through a permeable porous medium. The problem was first modeled into a coupled system of nonlinear partial differential equations of conservation of mass, momentum and nanoparticle concentration. The system of coupled nonlinear boundary layer partial differential equations governing the flowing fluid momentum and heat transfer characteristics are reduced to a new simplified coupled nonlinear system of ordinary differential equations by means of a suitable similarity transformation. The transformed set of nonlinear coupled ordinary differential equations is than solved numerically by means of the fourth order numerical scheme the Runge-Kutta shooting method. The effects of important involved parameters that control the flow field and heat transfer characteristics, that is the viscosity parameter, the convection parameter, the Porosity parameter, the Prandtl number and the Lewis number have been obtained and discussed. Numerical solutions for velocity and temperature are sketched and graphically analyzed. The graphical results observed are indicating that by increasing the values of the non-dimensional viscosity parameter, the dimension less fluid flow profile increases, while for increasing values of the nanoparticles Brownian motion parameter, the nanoparticle concentration profile increases.
\end{abstract}

Keywords: Boundary Layer Flow, Permeable Medium, Porous Plate

\section{Introduction}

The term "nanofluid" refers to a liquid containing a suspension of submicronic solid particles (nanoparticles). The term nanofluid was introduced by Choi [1] and this area which has attracted the attention of the researchers. The characteristic feature of nanofluids is thermal conductivity enhancement, a phenomenon observed by Masuda et al. [2]. The flow due to porous sheet has numerous applications due to its practical and cost related advantages, it has been extensively used in many engineering fields and industrial manufacturing processes such as the aerodynamic extrusion of plastic sheets, bundle wrapping, hot rolling, extrusion of sheet material, wire rolling etc. Vadasz [3] studied the concept of porous medium and explore some emerging topics in heat and mass transfer and developed some useful results on that medium. Ingham and Pop [4] studied the phenomena of transportation of fluid and heat in porous medium. Cheng [5] investigated problem of natural convection heat transfer of non-Newtonian fluids in porous media from a vertical cone under mixed thermal boundary conditions and developed useful underlying results with the help of numerical techniques. Ahmad and Pop [6] studied important results and properties of mixed convection boundary layer flow from a vertical flat plate embedded in a porous medium filled with nanofluids. Cheng [7] further discuss about on soret and dufour effects on heat and mass transfer by natural convection from a vertical truncated cone in a fluidsaturated porous medium with variable wall temperature and concentration. Sheikholeslami et al. [8] studied the effects of heat transfer in flow of nanofluids over a permeable stretching wall in a porous medium. Abbasi et al. [9] analyze the phenomenon which is based on Peristaltic transport of copper-water nanofluid saturating porous medium. Sheikholeslami et al. [10] further observed some 
basic results for the problem with numerical modeling for alumina nanofluid magnetohydrodynamic convective heat transfer in a permeable medium using Darcy law. Hassan et al. [11] investigated the properties and results of convective heat transfer flow of nanofluid in a porous medium over wavy surface. Reddy et al. [12] studied the influence of chemical reaction, radiation and rotation on MHD nanofluid flow past a permeable flat plate in porous medium. Few other related works regarding the boundary layer flows, fluids containing nanoparticles and heat transfer analysis are cited in [13-16].

A boundary layer is the layer of fluid in the immediate vicinity of a bounding surface where the effects of viscosity are significant [17-19]. The present paper studies the problem of boundary layer of a nanofluid through a porous sheet. The governing equations are transformed into nonlinear coupled ordinary differential equations which depends on the viscosity ratio parameter $\Lambda$, Prandtl number Pr. The obtained nonlinear coupled ordinary differential equations are solved numerically using shooting iteration technique [20-21]. The velocity, temperature, and concentration distributions are discussed and presented graphically, and the skin-friction coefficient, the surface heat, and mass transfer rate at the sheet are investigated.

\section{Mathematical Formulation}

We consider a two-dimensional problem. Consider a steady two-dimensional flow of an incompressible viscous nanofluid caused by a porous medium. A flat plate is called porous if fluid can be entered and/or leave the control volume through the surface of the plate. We select a coordinate frame in which the $x$-axis is aligned vertically upwards. We consider a vertical plate at $y=0$. The fluid is occupying the region $x>0$. If the plate is such that fluid can enter from the region $x<0$ into $x>0$ (injection/blowing flow) or fluid can cross from $x>0$ and enters $x>0$ (suction flow) then plate is termed as porous. The velocity for the blowing case is taken positive and for that of the suction is negative. The uniform temperature of the plate raised to $T_{w}\left(>T_{\infty}\right)$, which is thereafter maintained constant, where $T_{w}$ is temperature at the wall and $T_{\infty}$ is temperature far away from the plate

For the problem of fluid flowing over a porous plate the velocity flied is of the form

$$
V=(u(x, y), v(x, y), 0), T=T(x, y), C=C(x, y)
$$

Then the governing equations of conservation of mass, momentum, heat transfer and concentration are [22-25]

$$
\begin{gathered}
\frac{\partial u}{\partial x}+\frac{\partial v}{\partial y}=0 \\
u \frac{u}{\partial x}+v \frac{\partial u}{\partial y}=\tilde{v} \frac{\partial^{2} u}{\partial y^{2}}+g \beta\left(T-T_{\infty}\right)-\frac{\tilde{v} \phi_{p}}{k_{0}} u
\end{gathered}
$$

$$
\begin{gathered}
u \frac{\partial T}{\partial x}+v \frac{\partial T}{\partial y}= \\
\alpha \frac{\partial^{2} T}{\partial y^{2}}+\frac{\rho^{*} c_{p}^{*}}{\rho c_{p}}\left(D_{T} \frac{\partial T}{\partial y} \frac{\partial C}{\partial y}+\frac{D_{T}}{T_{\infty}}\left(\frac{\partial T}{\partial y}\right)^{2}\right) \\
u \frac{\partial C}{\partial x}+v \frac{\partial C}{\partial y}=D_{B} \frac{\partial^{2} C}{\partial y^{2}}+\frac{D_{T}}{T_{\infty}} \frac{\partial^{2} T}{\partial y^{2}}
\end{gathered}
$$

Subject to the boundary conditions

$$
\begin{gathered}
v=v_{w}, T=T_{w}(x), C=C_{w}(x) \text { at } y=0 \\
u \rightarrow \infty, T \rightarrow T_{\infty}, C \rightarrow C_{\infty} \text { as } y \rightarrow \infty
\end{gathered}
$$

Where $(u, v)$ are the velocity components along $(x, y)$ axes, $\tilde{v}$ is the kinematic viscosity of the base fluid, $T$ is temperature of the base fluid, $T_{w}$ is the surface temperature of the sheet, $c_{p}^{*}$ is the nanoparticles effective heat, the Brownian diffusion constant is $D_{B}$, the thermophoretic diffusion constant is $D_{T}, \phi_{p}$ is the porosity of permeable space, and $k_{0}$ is the penetrability of permeable space.

Introduce the following similarity transformations [26-28] in Equations (8-10)

$$
\begin{gathered}
\eta=\frac{y}{x} \sqrt{\frac{u_{o}}{2 v L}}, \quad \psi=\sqrt{2 v u_{o} L} f(\eta) \\
\theta(\eta)=\frac{T-T_{\infty}}{T_{w}-T_{\infty}}, \varphi(\eta)=\frac{C-C_{\infty}}{C_{w}-C_{\infty}}, \\
u=\frac{\partial \psi}{\partial y}=u_{o} f^{\prime}, \quad v=-\frac{\partial \psi}{\partial x}=-\sqrt{\frac{v u_{o}}{2 L}}\left(f-\eta f^{\prime}\right)
\end{gathered}
$$

Apply these similarity transformations over Equations (2-7) to reduce the partial differential equations into a new set of ordinary differential equations of the form

$$
\begin{gathered}
\Lambda f^{\prime \prime \prime}+f f^{\prime \prime}-2 f^{\prime 2}+2 \lambda_{t} \theta-k_{p} f^{\prime}=0 \\
\theta^{\prime \prime}+\operatorname{Pr}\left(f \theta^{\prime}-f^{\prime} \theta\right)+N_{b} \theta^{\prime} \varphi^{\prime}+N_{t} \theta^{2}=0 \\
\varphi^{\prime \prime}+\operatorname{Le}\left(f \varphi^{\prime}-f^{\prime} \varphi\right)+\left(\frac{N_{t}}{N_{b}}\right) \theta^{\prime \prime}=0
\end{gathered}
$$

Where $\Lambda$ is the viscosity ratio parameter, $\lambda_{t}$ is the convection parameter, $k_{p}$ is the porosity parameter, $\operatorname{Pr}$ is the Prandtl number $L e$ is the non-dimensional Lewis number, $N_{b}=\frac{(\rho c) D_{B}\left(C_{w}-C_{\infty}\right)}{v(\rho c)_{f}}$ is the Brownian motion parameter and $\quad N_{t}=\frac{(\rho c)_{p} D_{T}\left(T_{w}-T_{\infty}\right)}{v T_{\infty}(\rho c)_{f}}$ is he Thermophoresis parameter. The reduced boundary conditions are

$$
f=s, f^{\prime}=1, \theta=1, \varphi=1 \text { at } \eta=0
$$




$$
f^{\prime}=0, \theta=0, \varphi=10 \text { as } \eta \rightarrow \infty
$$

\section{Solution of the Problem}

The system of ordinary differential equations in Equations (11-13) subject to the boundary conditions in Equations (1415 ) is solved numerically using the fourth order shooting method [29-31]. The graphical behaviors of the different important involved parameters are graphically presented in Figures (1-6). Figure 1 represents the behavior of convection parameter $\lambda_{t}$ over the non-dimensional velocity profile $f^{\prime}(\eta)$. From the graph it is observed that with an increase in the convection parameter $\lambda_{t}$ the non-dimensional velocity profile $f^{\prime}(\eta)$ increases. Figure 2 denotes the behavior of viscosity ratio parameter $\Lambda$ over the non-dimensional velocity profile $f^{\prime}(\eta)$. From the graph it is noted that with an increase in the viscosity ratio parameter $\Lambda$ the non-dimensional velocity profile $f^{\prime}(\eta)$ increases. Figure 3 represents the behavior of Prandtl numbers $P r$ over the non-dimensional temperature profile $\theta(\eta)$. From the graph it is observed that with an increase in Prandtl numbers $\operatorname{Pr}$ the non-dimensional temperature profile $\theta(\eta)$ decreases. Figure 4 represents the behavior of thermophoresis parameter $N_{t}$ over the nondimensional temperature profile $\theta(\eta)$. From the graph it is observed that with an increase in the thermophoresis parameter $N_{t}$ the non-dimensional temperature profile $\theta(\eta)$ increases. Figure 5 represents the behavior of thermophoresis parameter $N_{b}$ over the non-dimensional concentration profile $\varphi(\eta)$. From the graph it is observed that with an increase in the thermophoresis parameter $N_{b}$ the non-dimensional temperature profile $\varphi(\eta)$ decreases. Figure 6 represents the behavior of thermophoresis parameter $N_{t}$ over the nondimensional concentration profile $\varphi(\eta)$. From the graph it is observed that with an increase in the thermophoresis parameter $N_{t}$ the non-dimensional temperature profile $\varphi(\eta)$ increases.

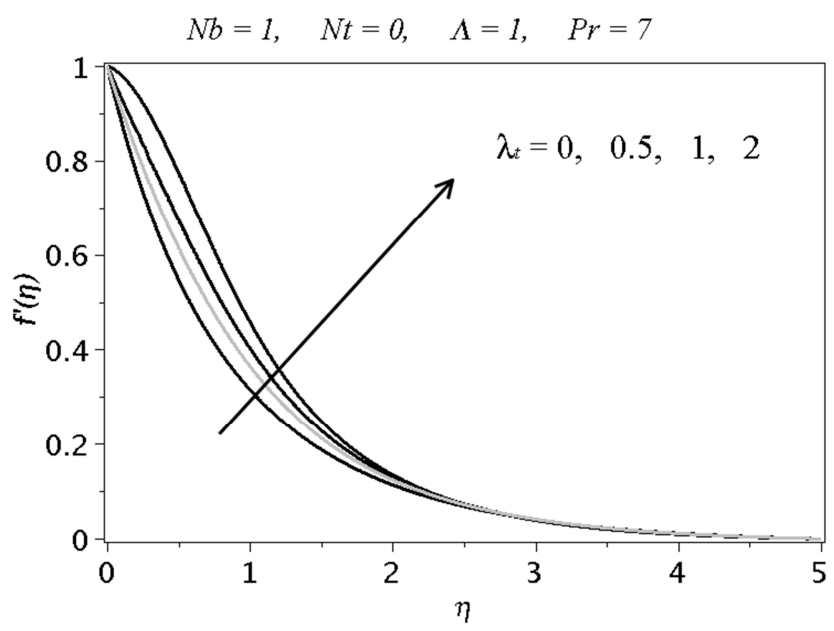

Figure 1. Velocity profile $f^{\prime}(\eta)$ for different values of $\lambda_{t}$.

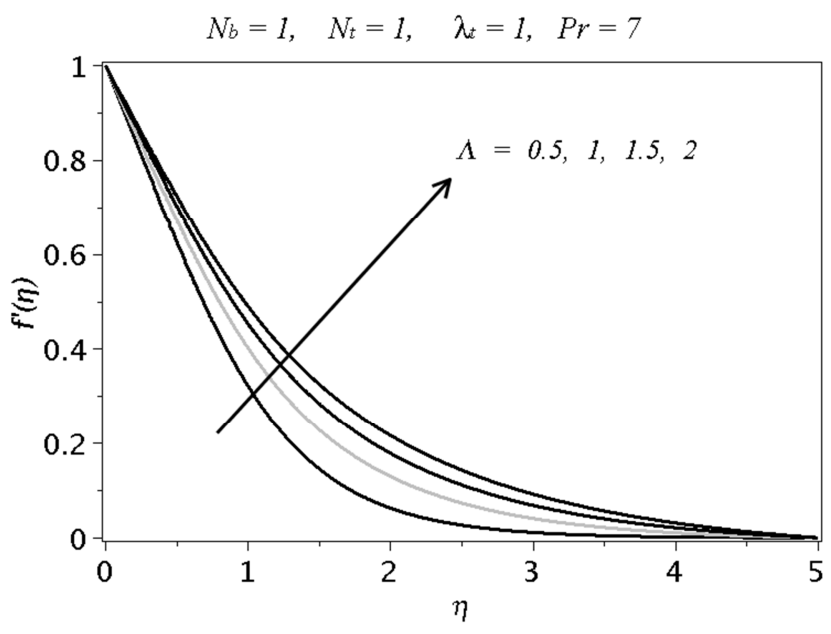

Figure 2. Velocity profile $f^{\prime}(\eta)$ for different values of $\Lambda$.

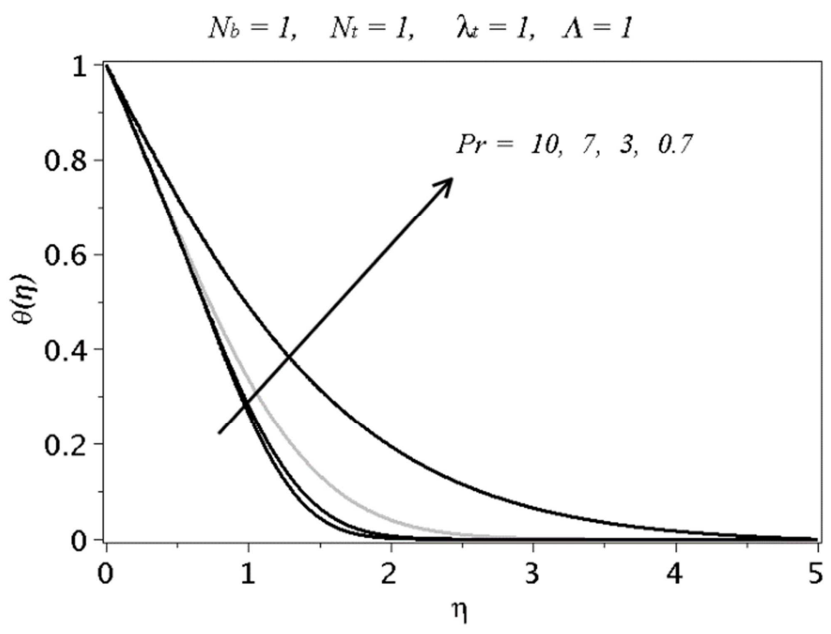

Figure 3. Temperature profile $f^{\prime}(\eta)$ for different values of $\mathrm{Pr}$.

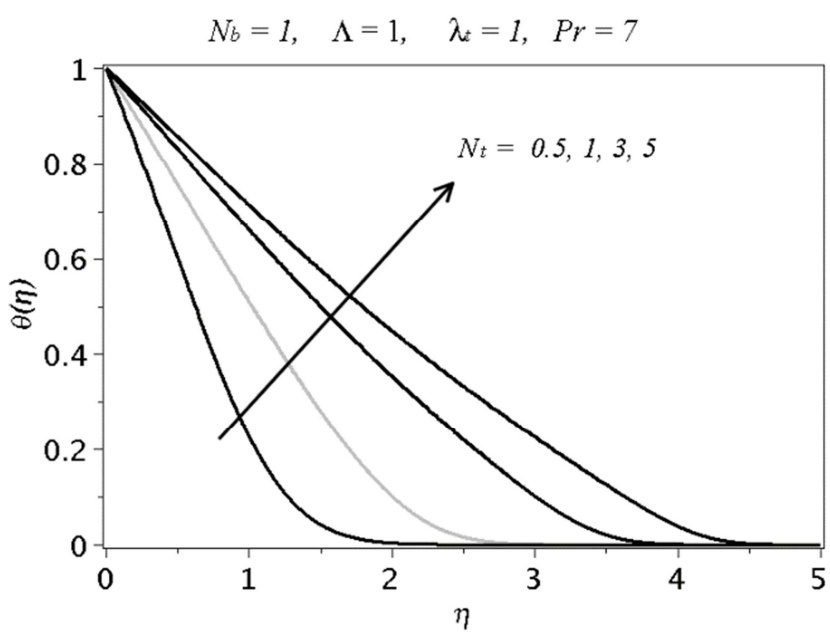

Figure 4. Temperature profile $\theta(\eta)$ for different values of $N_{t}$. 


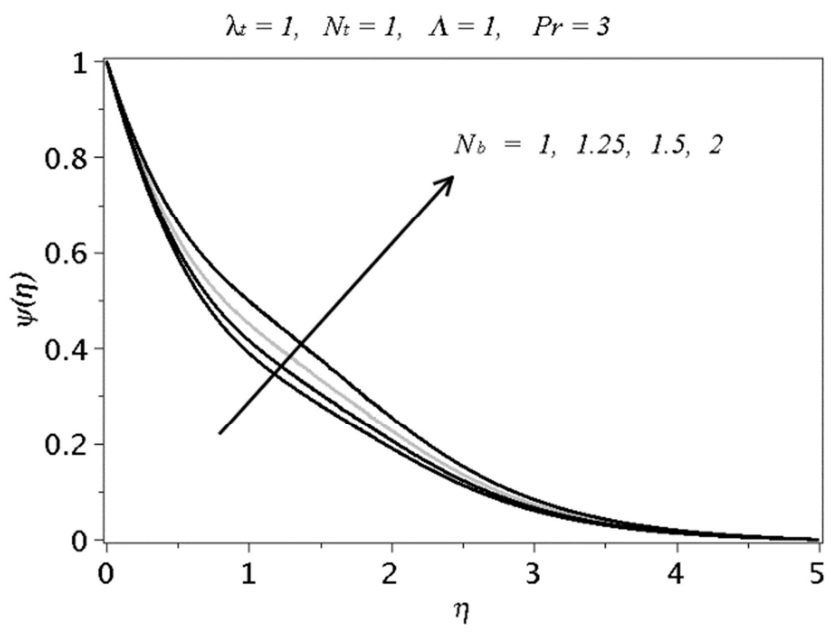

Figure 5. Temperature profile $\varphi(\eta)$ for different values of $N_{b}$.

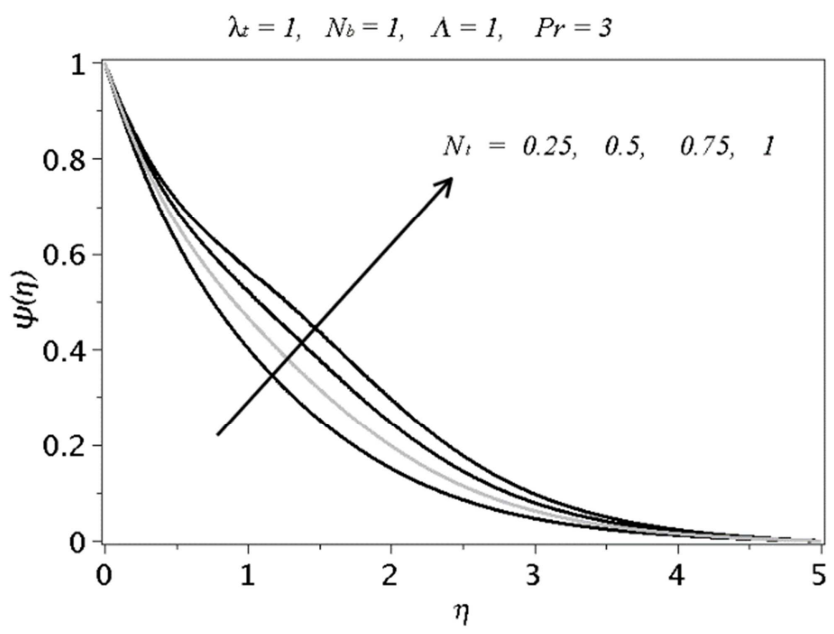

Figure 6. Temperature profile $\varphi(\eta)$ for different values of $N_{t}$.

\section{Conclusion}

The main conclusions obtained from the analysis are:

1) With increase in the viscosity parameter, the velocity profile increases.

2) With increase in the Prandtl number, the temperature profile decreases.

3) With increase in the Brownian motion parameter, the nanoparticle concentration profile increases.

\section{References}

[1] S. U. S. Choi, "Enhancing thermal conductivity of fluids with nanoparticle," in Developments and Applications of NonNewtonian Flows, D. A. Siginer and H. P. Wang, Eds., vol. 231, pp. 99-105, ASME, New York, NY, USA, 1995.

[2] H. Masuda, A. Ebata, K. Teramea, and N. Hishinuma, "Altering the thermal conductivity and viscosity of liquid by dispersing ultra-fine particles," Netsu Bussei, Vol. 4, No. 4, pp. 227-233, 1993.

[3] P. Vadasz, Emerging Topics in Heat and Mass Transfer in Porous Media, Springer, New York, 2008.
[4] D. B. Ingham, I. Pop (Eds.), Transport Phenomena in Porous Media, Vol. III, Elsevier, Oxford, 2005.

[5] Cheng C-Y, Natural convection heat transfer of nonNewtonian fluids in porous media from a vertical cone under mixed thermal boundary conditions. Int. Comm. Heat Mass Transfer 36: 693-697, 2009.

[6] Ahmad S, Pop I, Mixed convection boundary layer flow from a vertical flat plate embedded in a porous medium filled with nanofluids. Int. Comm. Heat Mass Transfer 37: 987-991, 2010.

[7] Cheng C-Y, Soret and Dufour effects on heat and mass transfer by natural convection from a vertical truncated cone in a fluid-saturated porous medium with variable wall temperature and concentration. Int. Comm. Heat Mass Transfer 37: 1031-1035, 2010.

[8] Sheikholeslami, M., R. Ellahi, H. R. Ashorynejad, G. Domairry, and T. Hayat. "Effects of heat transfer in flow of nanofluids over a permeable stretching wall in a porous medium." Journal of Computational and Theoretical Nanoscience 11, no. 2 (2014): 486-496.

[9] Abbasi, F. M., T. Hayat, and B. Ahmad. "Peristaltic transport of copper-water nanofluid saturating porous medium." Physica E: Low-dimensional Systems and Nanostructures 67 (2015): 47-53.

[10] Sheikholeslami, M., S. A. Shehzad, Zhixiong Li, and Ahmad Shafee. "Numerical modeling for alumina nanofluid magnetohydrodynamic convective heat transfer in a permeable medium using Darcy law." International Journal of Heat and Mass Transfer 127 (2018): 614-622.

[11] Hassan, M., M. Marin, Abdullah Alsharif, and R. Ellahi. "Convective heat transfer flow of nanofluid in a porous medium over wavy surface." Physics Letters A 382, no. 38 (2018): 2749-2753.

[12] Reddy, JV Ramana, V. Sugunamma, N. Sandeep, and C. Sulochana. "Influence of chemical reaction, radiation and rotation on MHD nanofluid flow past a permeable flat plate in porous medium." Journal of the Nigerian Mathematical Society 35 , no. 1 (2016): 48-65.

[13] S. Nadeem, Abdul Rehman, K. Vajravelu, Jinho Lee, Changhoon Lee, Axisymmetric stagnation flow of a micropolar nanofluid in a moving cylinder, Mathematical Problems in Engineering, Volume 2012, Article ID 378259 .

[14] Abdul Rehman, S. Nadeem, Mixed convection heat transfer in micropolar nanofluid over a vertical slender cylinder, Chin. Phy. Lett. 29 (12) (2012) 124701-5.

[15] S. Nadeem, Abdul Rehman, Changhoon Lee, Jinho Lee, Boundary layer flow of second grade fluid in a cylinder with heat transfer, Mathematical Problems in Engineering, Volume 2012, Article ID 640289.

[16] S. Nadeem, Abdul Rehman, Mohamed Ali, The boundary layer flow and heat transfer of a nanofluid over a vertical slender cylinder, J. NanoEngineering and NanoSystems (2012) $1-9$.

[17] S. Nadeem, Abdul Rehman, Axisymmetric stagnation flow of a nanofluid in a moving cylinder, Comp. Math. Mod. 24 (2) (2013) 293-306. 
[18] Abdul Rehman, S. Nadeem, M. Y. Malik, Stagnation flow of couple stress nanofluid over an exponentially stretching sheet through a porous medium, J. Power Tech. 93 (2) (2013) 122132 .

[19] Abdul Rehman, S. Nadeem, M. Y. Malik, Boundary layer stagnation-point flow of a third grade fluid over an exponentially stretching sheet, Braz. J. Che. Eng. 30 (3) (2013) 611-618.

[20] Abdul Rehman, S. Nadeem, Heat transfer analysis of the boundary layer flow over a vertical exponentially stretching cylinder, Global J. Sci. Fron. Res. 13 (11) (2013) 73-85.

[21] M. Y. Malik, M. Naseer, S. Nadeem, Abdul Rehman, The boundary layer flow of Casson nanofluid over a vertical exponentially stretching cylinder, Appl. NanoSci. DOI: 10.1007/s13204-012-0267-0.

[22] Abdul Rehman, S. Nadeem, S. Iqbal, M. Y. Malik, M. Naseer, Nanoparticle effect over the boundary layer flow over an exponentially stretching cylinder, J. NanoEngineering and NanoSystems (2014) 1-6.

[23] M. Y. Malik, M. Naseer, S. Nadeem, Abdul Rehman, The boundary layer flow of hyperbolic tangent fluid over a vertical exponentially stretching cylinder, Alexandria Eng. J., 53 (2014) 747-750.

[24] M. Y. Malik, M. Naseer, Abdul Rehman, Numerical study of convective heat transfer on the Power Law fluid over a vertical exponentially stretching cylinder, App Comp Math, 4 (5), (2015) 346-350.
[25] Abdul Rehman, R. Bazai, S. Achakzai, S. Iqbal, M. Naseer, Boundary Layer Flow and Heat Transfer of Micropolar Fluid over a Vertical Exponentially Stretched Cylinder, App Comp Math, 4 (6) (2015) 424-430.

[26] Abdul Rehman, G. Farooq, I. Ahmed, M. Naseer, M. Zulfiqar, Boundary Layer Stagnation-Point Flow of Second Grade Fluid over an Exponentially Stretching Sheet, American J App Math Stat, 3 (6) (2015) 211-219.

[27] Abdul Rehmana, S. Achakzai, S. Nadeem, S. Iqbal, Stagnation point flow of Eyring Powell fluid in a vertical cylinder with heat transfer, Journal of Power Technologies 96 (1) (2016) $57-62$.

[28] Abdul Rehman, Saleem Iqbal, Syed Mohsin Raza, Axisymmetric Stagnation Flow of a Micropolar Fluid in a Moving Cylinder: An Analytical Solution, Fluid Mechanics, 2 (1) (2016) 1-7.

[29] Naheeda Iftikhar, Abdul Rehman, Peristaltic flow of an Eyring Prandtl fluid in a diverging tube with heat and mass transfer, International Journal of Heat and Mass Transfer 111 (2017) 667-676.

[30] Abdul Rehman, Naveed Sheikh, Boundary Layer StagnationPoint Flow of Micropolar Fluid over an Exponentially Stretching Sheet, International Journal of Fluid Mechanics \& Thermal Sciences, 2017; 3 (3): 25-31.

[31] Haroon Rasheed, Abdul Rehman, Naveed Sheikh, Saleem Iqbal, MHD Boundary Layer Flow of Nanofluid over a Continuously Moving Stretching Surface, Applied and Computational Mathematics, 2017; 6 (6): 265-270. 